\title{
THREEFOLDS WITH NEGATIVE KODAIRA DIMENSION AND POSITIVE IRREGULARITY
}

\author{
MAURO BELTRAMETTI AND PAOLO FRANCIA
}

\section{§ 0. Introduction}

The purpose of this paper is to study threefolds $X$, with negative Kodaira dimension $\kappa(X)$ and positive irregularity $q(X)$, defined over the complex field $\boldsymbol{C}$.

In Section 1 we recall some definitions and preliminary results. The main statements are contained in Section 2. We prove the following:

I) Assume the Euler-Poincaré characteristic $\chi\left(\mathcal{O}_{X}\right)$ is positive. Then $X$ is birationally equivalent to a conic bundle on a surface $S$ such that $\kappa(S) \geqslant 0$.

II) Suppose $\chi\left(\mathcal{O}_{X}\right)<0$. Then there exist a projective nonsingular curve $C$ of positive genus and a morphism $X \rightarrow C$ such that the general fibre is a rational surface.

Statement I) also follows by combining some results due to T. Mabuchi and K. Ueno. Precisely, $X$ is uniruled whenever $q(X)>0$, as pointed out by $\mathrm{K}$. Ueno in [U2]. Using this fact, then the assert can be obtained from a more general result contained in [M], that requires a rather hard and lengthy proof. Our argument is more direct and it does not use the uniruledness of $X$.

Statement II) gives also the converse of another result due to $\mathrm{T}$. Mabuchi (see [M], 2. 3. 2.).

In case $\chi\left(\mathcal{O}_{X}\right)=0$ then $X$ falls into item I) or II) according to whether $H^{0}\left(X, S^{12}\left(\Omega_{X}^{2}\right)\right)$ has positive or zero dimension.

Finally, in Section 3 a more explicit description of threefolds belonging to family II) is given by using the Enriques-Iskovskih classification of minimal rational surfaces (see [I], Theorem 1). Precisely, we show that there exists a birational minimal model $\tilde{X}$ of $X$ such that:

a) $\tilde{X}=C \times P^{2}$, or 
b) $\tilde{X}$ is a conic bundle on a surface birationally equivalent to $C \times$ $P^{1}$, or

c) there exists a morphism $\tilde{X} \rightarrow C$ whose generic fibre $S$ (in the sense of Grothendieck) is a Del Pezzo surface with the Picard group $\operatorname{Pic}(S) \simeq Z$ generated by the anticanonical sheaf $\omega_{S}^{-1}$ and $1 \leqslant \omega_{S}^{2} \leqslant 6$.

We would like to thank S. Mori for some helpful conversation.

\section{$\S 1$. Notations, definitions, and preliminary results}

By variety we mean a complete nonsingular algebraic variety defined over the complex field. For all coherent sheafs $\mathscr{F}$ on a $d$-dimensional variety $V$ we denote by $h^{i}(\mathscr{F})$ the dimension of the complex vector space $H^{i}(V, \mathscr{F}), i \geqslant 0$. Moreover we call irregularity of $V$ the integer $q(V)=$ $h^{1}\left(\mathcal{O}_{V}\right)$ and we note by $\chi\left(\mathcal{O}_{V}\right)=\sum_{i=0}^{d}(-1)^{i} h^{i}\left(\mathcal{O}_{V}\right)$ the Euler-Ponicaré characteristic of $V$. The $m$-plurigenus of $V, m$ positive integer, is $p_{m}(V)=$ $h^{0}\left(\omega_{V}^{\otimes m}\right)$, where $\omega_{V}$ is the canonical sheaf. Finally, for all $p, 0 \leqslant p \leqslant d$, we denote by $S^{r}\left(\Omega_{V}^{p}\right)$ the $r$-symmetric tensor of the sheaf of regular $p$ forms $\Omega_{V}^{p}$.

Let $f: V \rightarrow W$ be a surjective morphism with connected fibres of a $n$-dimensional variety $V$ over a $m$-dimensional variety $W$. Iitaka conjectured the following inequality for the Kodaira dimension $k($.$) to be$ true (see [U1], IV, §11):

ConJeCtuRe $C_{n, m} . \quad \kappa(V) \geqslant \kappa(W)+\kappa\left(V_{w}\right)$ where $V_{w}=f^{-1}(w), w$ closed point in $W$, is a general fibre of $f$. As proved in [V1], [V2], conjecture $C_{n, n-1}$ holds for $n \geqslant 2$, while in the case $n=3$ also conjecture $C_{3,1}$ is true.

From now on, by threefold we mean a nonsingular projective variety of dimension 3 .

Definition. Let $V$ be a threefold. We say that $V$ is a conical fibration if there exist a projective surface $Y$ and a rational map $h: V \rightarrow Y$ whose general fibre is a rational curve.

In the sequel we frequently use the following results contained in $[\mathrm{M}], 2.3 .1,2.3 .2$.

Proposition 1.1 (Mabuchi). Let $h: V \rightarrow Y$ be a conical fibration. Then $h^{*}: H^{0}\left(Y, S^{m}\left(\Omega_{Y}^{p}\right)\right) \rightarrow H^{0}\left(V, S^{m}\left(\Omega_{V}^{p}\right)\right)$ is an isomorphism for all positive integers $m$ and $p=0,1,2$.

Proposition 1.2 (Mabuchi). Let $X$ (resp. C) be a threefold (resp. a 
nonsingular projective curve) and $f: X \rightarrow C$ be a surjective morphism whose general fibre is an irreducible nonsingular rational surface. Then for all positive integers $m, p$ we have the isomorphism $f^{*}: H^{\circ}\left(C, S^{m}\left(\Omega_{C}^{p}\right)\right) \simeq$ $H^{0}\left(X, S^{m}\left(\Omega_{X}^{p}\right)\right)$.

Definition. We say that a threefold $X$ is a conic bundle if there exist a nonsingular projective surface $S$ and a morphism $f: X \rightarrow S$ such that all fibres are isomorphic to conics.

A connection between conical fibrations and conic bundles is given by the following result contained in [Z].

TheOREm 1.3 (Zagorskii). For any conical fibration $(V, Y, h)$ there exists a conic bundle $(X, S, f)$ together with a birational morphism $g: S \rightarrow Y$ and a birational map $\tilde{g}: X \rightarrow V$ such that the following diagram commutes:

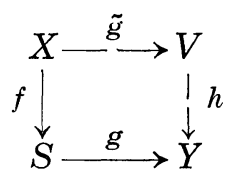

We frequently use results on surfaces' classification, for which we refer to [B].

\section{§2. Birational structure of threefolds with $\kappa(X)<0, q(X)>0$}

Our aim is to prove the following

TheOREM 2.1 Let $X$ be a threefold with $\kappa(X)<0, q(X)>0$. Then we have:

I) $X$ is birationally equivalent to a conic bundle on a surface $S$ such that $\kappa(S) \geqslant 0$, or

II) The image of the Albanese mapping $\alpha: X \rightarrow \operatorname{Alb}(X)$ is a nonsingular curve and the general fibre of $\alpha$ is a rational surface.

Furthermore if $\chi\left(\mathcal{O}_{X}\right)>0$, or $\chi\left(\mathcal{O}_{X}\right)=0$ and $h^{0}\left(S^{12}\left(\Omega_{X}^{2}\right)\right)>0$, then $X$ belongs to family I); while, whenever $\chi\left(\mathcal{O}_{X}\right)<0$, or $\chi\left(\mathcal{O}_{X}\right)=0$ and $h^{0}\left(S^{12}\left(\Omega_{X}^{2}\right)\right)$ $=0$, then $X$ belongs to family II).

Proof. Consider the Stein factorization of the Albanese mapping $\alpha$ :

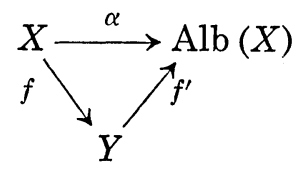


The morphism $f$ has connected fibres and $f^{\prime}$ is finite. By well known general facts we have $\kappa(Y) \geqslant 0, \operatorname{dim} Y \leqslant 2$ (see [U1], II $\S 6$, IV $\S 10$ ). Suppose $\operatorname{dim} Y=2$. Then $C_{3,2}$ gives

$$
\kappa(X) \geqslant \kappa(F)+\kappa(Y),
$$

$F$ general fibre of $f$. Thus $k(F)<0$, i.e. $F \simeq \boldsymbol{P}^{1}$. Hence we are done by use of Zagorskii result.

Therefore we can suppose that the image of the Albanese mapping is a curve $C$, in which case $C$ is nonsingular of genus $p_{q}(C)=q(X)$ and the fibres of $\alpha: X \rightarrow C$ are connected ([U1], 9.19).

Again the general fibre $F$ of $\alpha: X \rightarrow C$ has negative Kodaira dimension by $C_{3,1}$, so that $F$ is a ruled surface. As the morphism $\alpha$ is flat (see $[\mathrm{H}]$, III, 9.7) it follows that all general fibres $F$ have the same irregularity. In fact $p_{g}(F)=0$ and the Euler-Poincaré characteristic does not depend on $F([\mathrm{H}], \mathrm{III}, 9.9)$.

First, suppose the irregularity $q(F)$ to be zero. Then $F$ is a rational surface, so that $X$ belongs to family II).

Thus, from now on, we can assume the irregularity $q(F)$ to be positive. Then there exist a nonsingular projective curve $B$ with $p_{g}(B)=q(F)$ and a morphism $\varphi: F \rightarrow B$ whose general fibre is isomorphic to $\boldsymbol{P}^{1}$.

We fix now a pair $F, \Gamma$, where $F$ is a nonsingular fibre of $\alpha: X \rightarrow C$ and $\Gamma \simeq \boldsymbol{P}^{1}$ is a fibre of the morphism $\varphi: F \rightarrow B$. Consider the exact sequence of normal bundles:

$$
0 \longrightarrow \mathscr{N}_{\Gamma / F} \longrightarrow \mathscr{N}_{\Gamma / X} \longrightarrow \mathscr{N}_{F / X} \otimes \mathcal{O}_{\Gamma} \longrightarrow 0 \text {. }
$$

One has $\Gamma \cdot F=0$. Moreover $\left(\Gamma^{2}\right)_{F}=0$. This means that $\mathscr{N}_{F / X} \otimes \mathcal{O}_{\Gamma} \simeq$ $\mathscr{N}_{\Gamma / F} \simeq \mathcal{O}_{\Gamma}$ and the sequence becomes

$$
0 \longrightarrow \mathcal{O}_{\Gamma} \longrightarrow \mathscr{N}_{\Gamma / X} \longrightarrow \mathcal{O}_{\Gamma} \longrightarrow 0 \text {. }
$$

Therefore

$$
h^{0}\left(\mathscr{N}_{\Gamma / X}\right)=2, \quad h^{1}\left(\mathscr{N}_{\Gamma / X}\right)=0 .
$$

Then from a general theorem contained in [FGA], exp. 221, the Hilbert scheme $\operatorname{Hilb}(\Gamma, X)$ is a surface which is smooth at the point $s_{0}$ representing $\Gamma$. Let $S_{0}$ be the irreducible component of $\operatorname{Hilb}(\Gamma, X)$ containing $s_{0}$ and let $F(X)$ be the universal family of curves of $X$ parametrized by $S_{0}$. Look to the commutative diagram 


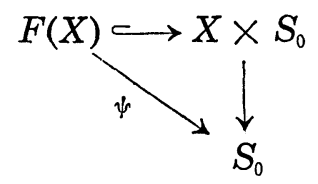

For all closed points $s \in S_{0}$ one has $\psi^{-1}(s)=\left(\Gamma_{s}, s\right)$ where $\Gamma_{s}$ is the curve of $X$ corresponding to $s$.

Moreover $F(X)$ is nothing but the set of the pairs $(x, s) \in X \times S_{0}$ such that $x \in \Gamma_{s}$.

Now let $U, V_{0}$ be the open sets of $C, S_{0}$ corresponding to the nonsingular fibres of the morphisms $\alpha, \psi$ respectively. Note that for all points $s \in V_{0}$ the curve $\Gamma_{s}$ is rational since $\psi$ is flat. Put

$$
F(X)_{0}=F(X) \cap\left(\alpha^{-1}(U) \times V_{0}\right) .
$$

To proceed further, the following claim is needed.

Claim. a) For every $(x, s) \in F(X)_{0}$ the curve $\Gamma_{6}$ is contained in the fibre $F_{y}, y=\alpha(x)$ and $\left(\Gamma_{s}^{2}\right)_{F_{y}}=0$.

b) For all elements $\left(x_{1}, s_{1}\right),\left(x_{2}, s_{2}\right) \in F(X)_{0}$ such that $\alpha\left(x_{1}\right)=\alpha\left(x_{2}\right)=y$ one has $\left(\Gamma_{1} \cdot \Gamma_{2}\right)_{F_{y}}=0$, where $\Gamma_{i}=\Gamma_{s_{i}}, i=1,2$.

Proof. (a) Since $\Gamma \cdot F=0$ and $F \stackrel{a}{\sim} F_{y}(\stackrel{a}{\sim}$ means algebraic equivalence of cycles in $X$ ) we have $\Gamma \cdot F_{y}=0$. As $\Gamma_{s} \stackrel{a}{\sim} \Gamma$, it follows $\Gamma_{s} \cdot F_{y}=0$. Moreover the curve $\Gamma_{s}$ contains $x$ so that $x \in \Gamma_{s} \cap F_{y}$. Therefore $\Gamma_{s} \subset F_{y}$.

Consider now the exact sequence

$$
0 \longrightarrow \mathscr{N}_{\Gamma_{s} / F_{y}} \longrightarrow \mathscr{N}_{\Gamma_{s} / X} \longrightarrow \mathscr{N}_{F_{y} / X} \otimes \mathcal{O}_{\Gamma_{s}} \longrightarrow 0
$$

Since $\Gamma_{s}$ is nonsingular the adjunction formula gives

$$
\operatorname{deg} \mathscr{N}_{\Gamma_{s} / X}=\operatorname{deg} \mathscr{N}_{\Gamma / X} \text {. }
$$

Hence $\operatorname{deg} \mathscr{N}_{\Gamma_{s} / X}=0$ in view of sequence (1). For $\Gamma_{s} \cdot F_{y}=0$ one has $\mathscr{N}_{F_{y} / X} \otimes \mathcal{O}_{\Gamma_{s}} \simeq \mathcal{O}_{\Gamma_{s}}$ so that $\operatorname{deg} \mathscr{N}_{F_{y} / X} \otimes \mathcal{O}_{\Gamma_{s}}=0$. It follows $\operatorname{deg} \mathscr{N}_{\Gamma_{s} / F_{y}}=0$, that is $\left(\Gamma_{s}^{2}\right)_{F y}=0$.

(b) Claim (a) gives

$$
\left(\Gamma_{1}^{2}\right)_{F_{y}}=\left(\Gamma_{2}^{2}\right)_{F_{y}}=0
$$

Moreover one has $h^{0}\left(\mathscr{N}_{\Gamma_{1} / F_{y}}\right)=h^{0}\left(\mathscr{N}_{\Gamma_{2} / F_{y}}\right)=1$. Then $\Gamma_{1}, \Gamma_{2}$ define two algebraic systems $\Phi_{1}, \Phi_{2}$ on $F_{y}$ of dimension 1 . Suppose now $\left(\Gamma_{1} \cdot \Gamma_{\mathrm{\varepsilon}}\right)_{F_{y}}=$ 
$n>0^{(*)}$ Fix $\Gamma_{1}$ and let $Z$ be the curve parametrizing $\Phi_{2}$. For all points $t \in \Gamma_{1}$ let $Z_{t}$ be the element of $\Phi_{2}$ determined by passing through $t$. So we get a morphism $\Gamma_{1} \rightarrow Z$, of degree $n$, such that $t$ goes into $Z_{t}$. Therefore $Z$ is rational being $\Gamma_{1} \simeq \boldsymbol{P}^{1}$. This means that $\Phi_{2}$ is just a 1-dimensional linear system (of rational curves). It follows that $F_{y}$ is a rational surface, hence $q\left(F_{y}\right)=0$ : contradiction. Then $n=0$.

q.e.d.

A consequence of the Claim is that the projection $\pi: F(X) \rightarrow X$ is a birational morphism. To see this, take $\left(x_{1}, s_{1}\right),\left(x_{2}, s_{2}\right) \in F(X)_{0}$ and suppose $\pi\left(x_{1}, s_{1}\right)=\pi\left(x_{2}, s_{2}\right)$, so that $x_{1}=x_{2}=x \in \Gamma_{s_{1}} \cap \Gamma_{s_{2}}$. Therefore Claim (b) implies $\left(\Gamma_{s_{1}} \cdot \Gamma_{s_{2}}\right)_{F_{y}}=0$, where $y=\alpha(x)$. Hence $\Gamma_{s_{1}}=\Gamma_{s_{2}}$ on $F_{y}$. This means that $\pi$ is injective on the open set $F(X)_{0}$, that is $\pi$ is a birational morphism.

Thus a rational map $h: X \rightarrow S_{0}$ is defined, whose general fibre $h^{-1}(s)$ is isomorphic to $\boldsymbol{P}^{1}$. In fact $h^{-1}(s)$ coincides by construction with the curve $\Gamma_{s}$ of $X$ corresponding to $s$. Therefore $\left(X, S_{0}, h\right)$ is a conical fibration, so we are done by use of Zagorskii result.

To conclude the proof of the first part of the statement it remains to see that $\kappa\left(S_{0}\right) \geqslant 0$. For this look again at the rational map $h: X-\rightarrow S_{0}$. The general fibre $h^{-1}(s)$ is contained in a fibre of $\alpha: X \rightarrow C$ by Claim (a). Then a rational map $\beta: S_{0} \rightarrow C$ is defined, such that the diagram

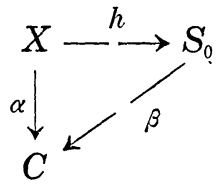

commutes. We can assume $h$ and $\beta$ to be morphisms: otherwise we consider birational models of $X$ and $S_{0}$. Suppose now $\kappa\left(S_{0}\right)<0$. Then conjecture $C_{2,1}$ implies that the general fibre of $\beta$ is isomorphic to $\boldsymbol{P}^{1}$. As before, let $F$ be the general fibre of $\alpha$. By base change $h$ induces a morphism $F \rightarrow \boldsymbol{P}^{1}$ whose general fibre is again isomorphic to $\boldsymbol{P}^{1}$ (see also [EGA], I, 3.4.8). Hence $F$ contains a 1-dimensional linear system of rational curves, so that $F$ is a rational surface. This is not the case by the assumption made $q(F)>0$. It follows $k\left(S_{0}\right) \geqslant 0$.

We prove now the last part of the statement. Suppose $X$ belongs

(*) Note that in this case, $\Gamma_{1}, \Gamma_{2}$ are not numerically equivalent on $F_{y}$ in view of relations (2), so that $\Phi_{1} \neq \Phi_{2}$. 
to family II). Then we get $h^{2}\left(\mathcal{O}_{X}\right)=0$ by Proposition 1.2. Therefore $\chi\left(\mathcal{O}_{X}\right)=1-q(X) \leqslant 0$. It follows that case II) does not occur when $\chi\left(\mathcal{O}_{X}\right)$ $>0$. Assume that $X$ belongs to family I). Hence one has $q(X)=q(S)$, $h^{0}\left(\Omega_{X}^{2}\right)=h^{0}\left(\omega_{S}\right)$ by Proposition 1.1, so that $\chi\left(\mathcal{O}_{X}\right)=\chi\left(\mathcal{O}_{S}\right)$. Moreover $\chi\left(\mathcal{O}_{S}\right) \geqslant 0$ since $\kappa(S) \geqslant 0$. Then $X$ belongs to family II) if $\chi\left(\mathcal{O}_{X}\right)<0$. In the case $\chi\left(\mathcal{O}_{X}\right)=0$ we consider $h^{0}\left(S^{12}\left(\Omega_{X}^{2}\right)\right)$. Again Propositions 1.1, 1.2 give the assert. Now Theorem 1.2 is completely proved. q.e.d.

Remark. Let $X$ be a threefold with negative Kodaira dimension. Then the condition $\chi\left(\mathcal{O}_{X}\right)<0$ is equivalent to $h^{2}\left(\mathcal{O}_{X}\right)=0, q(X) \geqslant 2$. In fact, assume $\chi\left(\mathcal{O}_{X}\right)<0$. By combining Theorem 2.1 and Proposition 1.2 we have $h^{2}\left(\mathcal{O}_{X}\right)=0$ so that $q(X) \geqslant 2$. The converse is clear. Hence in particular statement II) holds for a threefold $X$ whenever $\kappa(X)<0, h^{2}\left(\mathcal{O}_{X}\right)$ $=0$, and $q(X) \geqslant 2$. This gives the converse of Proposition 1.2. The following examples show that the condition $q(X) \geqslant 2$ can not left out. Consider $X=S \times P^{1}$ where $S$ is any surface such that $p_{g}(S)=0, p_{12}(S)$ $>0$ and $q(S)=1$ or 0 (f.e. let $S$ be a hyperelliptic surface or a Godeaux surface). Therefore $h^{2}\left(\mathcal{O}_{X}\right)=0, q(X)=1$ or 0 and $h^{0}\left(S^{12}\left(\Omega_{X}^{2}\right)\right)=p_{12}(S)$ by Proposition 1.1. Assume statement II of Theorem 2.1 holds. Then by Proposition 1.2 we get $p_{12}(S)=0$ : contradiction.

\section{§3. The case $\chi\left(\mathcal{O}_{X}\right)<0$}

We give here a birational classification of threefold belonging to family II) of Theorem 2.1. This is essentially founded on a result due to Iskovskih, contained in [I].

Proposition 3.1. Let $X$ be a threefold with $\kappa(X)<0, q(X)>0$. Suppose there exists a morphism $\alpha: X \rightarrow C$ such that $C$ is a nonsingular projective curve and the general fibre is a rational surface. Then $X$ is birationally equivalent to one of the following types of threefolds $\tilde{X}$ :

a) $\tilde{X}=C \times P^{2}$.

b) $\tilde{X}$ is a conic bundle on a surface $S$ birationally equivalent to $C \times P^{1}$

c) There exists a morphism $\tilde{X} \rightarrow C$ such that the generic fibre $S$ is a Del Pezzo surface with Pic $(S) \simeq Z$ generated by the anticanonical sheaf $\omega_{S}^{-1}$. Moreover $1 \leqslant \omega_{S}^{2} \leqslant 6$.

Proof. Let $K=C(C)$ be the function field of the curve $C$. First note that, as a consequence of Tsen's Theorem ([T]) the field $K$ satisfies 
the so called $C_{1}$ property, namely every hypersurface of degree $d \leqslant n-1$ in $\boldsymbol{P}_{K}^{n}$ contains a rational point over $K$. Denote by $F_{\eta}=X \otimes_{C} \operatorname{Spec} K$ the generic fibre of $\alpha: X \rightarrow C$. Since the general fibre of $\alpha$ is a rational surface over $C$, then one has $k\left(F_{\eta}\right)<0, q\left(F_{\eta}\right)=0$ (cf. [Ii], § 10.3, [H], III, 9.9 ), so that $F_{\eta}$ is a rational surface over $K$. Let $S$ be a minimal model of $F_{\eta}$. Hence $S$ belongs to one of the following families of surfaces (see [I], Theorem 1):

i ) $S=P_{K}^{2}$;

ii) $S=P_{K}^{1} \times P_{K}^{1}$ is a quadric in $P_{K}^{3}$, having $\operatorname{Pic}(S) \simeq Z$ generated by a hyperplane section;

iii) $S$ is a Del Pezzo surface with $\operatorname{Pic}(S) \simeq Z$ generated by the anticanonical sheaf $\omega_{S}^{-1}$.

iv) there exists a morphism $S \rightarrow B$ such that the generic fibre and the base curve $B$ are nonsingular curves of genus 0 .

It is not difficult to prove that there exist a threefold $\tilde{X}$ and a birational map $\tilde{X} \longrightarrow X$ such that the generic fibre of the composition $\tilde{X} \rightarrow C$ is isomorphic to the minimal model $S$ of $F_{\eta}$. Moreover the function field $K(S)$ of $S$ is isomorphic to the function field $C(X)$ of $X$ (see [EGA], I, 3.4.6).

As $K$ is $C_{1}$ one has $K\left(\boldsymbol{P}_{K}^{2}\right)=K\left(\boldsymbol{P}_{K}^{1} \times \boldsymbol{P}_{K}^{1}\right)=C\left(C \times \boldsymbol{P}^{2}\right)$. Thus in cases i), ii) $X$ is birationally equivalent to $C \times \boldsymbol{P}^{2}$.

Suppose now case iii) holds. We have to show $1 \leqslant \omega_{S}^{2} \leq 6$. This follows by [Mo], 3.5.2, where it is proved that the case $\omega_{S}^{2}=7$ does not occur, while if $\omega_{S}^{2}=8,9$ the Picard group Pic $(S)$ is not generated by the anticanonical sheaf.

Finally, consider case iv). Again, since $K$ is $C_{1}$, the base curve $B$ is isomorphic to $\boldsymbol{P}_{K}^{1}$. So we get an inclusion of the function fields $K\left(\boldsymbol{P}_{K}^{1}\right)$ $\hookrightarrow C(X)$ corresponding to the surjective morphism $S \rightarrow P_{K}^{1}$. This means that there exists a rational map

$$
X \rightarrow C \times P^{1}
$$

whose generic fibre is isomorphic to a conic. Then by use of Zagorskii result $X$ is birationally equivalent to a conic bundle. q.e.d.

To conclude, we summarize our results in the following table.

Remark. We acknowledge the Referee's remark according to which the same results in this paper are valid also for Kälher threefolds. In- 
Threefolds with $\kappa(X)<0, q(X)>0$.

\begin{tabular}{|c|c|c|c|}
\hline$\chi\left(\mathcal{O}_{x}\right)$ & $h^{0}\left(s^{12}\left(\Omega_{X}^{2}\right)\right)$ & Structure of $X$ & Birational structure of $X$ \\
\hline$>0$ & & $X \rightarrow S$ conical fibration, $\kappa(S) \geqslant 0$ & $X \rightarrow S$ conic bundle, $\kappa(S) \geqslant 0$ \\
\hline 0 & $>0$ & $X \rightarrow S$ conical fibration, $\kappa(S) \geqslant 0$ & $X \rightarrow S$ conic bundle, $\kappa(S) \geqslant 0$ \\
\hline 0 & 0 & As in the case $\chi\left(\mathcal{O}_{X}\right)<0$ & As in the case $\chi\left(\mathscr{O}_{X}\right)<0$ \\
\hline$<0$ & & $\begin{array}{l}\text { There exists morphism } X \rightarrow \mathrm{C} \text {, } \\
C \text { nonsingular curve of genus } \\
g(C)>0 \text { whose general fibre is a } \\
\text { rational surface. }\end{array}$ & $\begin{array}{l}\text { a) } C \times \boldsymbol{P}^{2} \\
\text { b) There exists a morphism } \\
X \rightarrow C, C \text { nonsingular curve } \\
\text { of genus } \mathrm{g}(C)>0 \text {, whose } \\
\text { generic fibre } S \text { is a Del } \\
\text { Pezzo surface with } \omega_{S}^{2}=1 \text {, } \\
\cdots, 6 \text { and } P i c(S) \simeq Z \text { gene- } \\
\text { rated by } \omega_{S}^{-1} \cdot \\
\text { c) } X \rightarrow S \text { conic bundle, } S \\
\text { birationally equivalent to } \\
C \times P^{1} \text {. }\end{array}$ \\
\hline
\end{tabular}

deed, the method of the proof works in this context too by virtue of Fujiki's theory.

\section{REFERENCES}

[B] A. Beauville, Surfaces algébriques complexes, Asterisque, 54 (1978).

[EGA] A. Grothendieck and J. Dieudonné, Éléments de Géométrie Algébrique, I. 166, Springer (1971), and II. Publ. I.H.E.S., 8 (1961).

[FGA] A. Grothendieck, Fondements de la Géométrie Algébrique, Sem. Bourbaki, 1957-62 (1962).

[H] R. Hartshorne, Algebraic geometry, Graduate Texts in Math., Springer, 52 (1977).

[I] V. A. Iskovskih, Minimal models of rational surfaces over arbitrary fields, Math. USSR-Izv., 14 (1980), 17-39.

[Ii] S. Iitaka, Algebraic geometry (An introduction to birational geometry of algebraic varieties), Graduate Texts in Math., Springer, 76 (1981).

[M] T. Mabuchi, Invariant $\beta$ and uniruled threefolds, preprint.

[Mo] S. Mori, Threefolds whose canonical bundles are not numerically effective, preprint.

[T] C. C. Tsen, Divisionsalgebren über Functionkörpern, Nachr. Wiss. Göttingen, 1933.

[U1] K. Ueno, Classification theory of algebraic varieties and compact complex spaces, Springer, 439 (1975).

[U2] - Classification of algebraic manifolds, Proc. Internat. Congress Math. Helsinki, (1978), 54-556.

[V1] E. Viehweg, Canonical divisors and the additivity of the Kodaira dimension for morphisms of relative dimension one, Compositio Math., 35 (1977), 197-223.

[V2] - Klassificationstheorie algebraischer Varietaten der Dimension drei, Compositio Math., 41 (1980), 361-400. 
[Z] A. Zagorskii, Three-dimensional conical fibrations, Matem. Zametki, 21, 6 (1977), 745-758; English transl. in Math. Notes, 21 (1977), 420-427.

Istituto Matematico

Universita di Genova

Via L. B. Alberti, 4-Genova

Italy 